\title{
On the Variational Problems without Having Desired Variational Symmetries
}

\author{
Mehdi Nadjafikhah, Saeed Dodangeh, and Parastoo Kabi-Nejad \\ School of Mathematics, Iran University of Science and Technology, Narmak, Tehran 1684613114, Iran \\ Correspondence should be addressed to Saeed Dodangeh; s_dodangeh@mathdep.iust.ac.ir
}

Received 14 January 2013; Revised 9 April 2013; Accepted 12 April 2013

Academic Editor: Peter Grabner

Copyright ( 2013 Mehdi Nadjafikhah et al. This is an open access article distributed under the Creative Commons Attribution License, which permits unrestricted use, distribution, and reproduction in any medium, provided the original work is properly cited.

We will have an attempt to present a method for constructing variational problems without having a desired one-parameter transformation group as a variational symmetry. For this, we use the notation of $\mu$-symmetry which was introduced by Giuseppe Gaeta and Paola Morando in 2004. Moreover, our given method enabled us to solve those constructed variational problems using $\mu$-symmetries.

\section{Introduction}

The applications of symmetry groups to problems arising in the calculus of variations have their origins in the late papers of Lie, which introduced the subject of "integral invariants" [1]. Lie showed how the symmetry group of a variational problem can be readily computed based on an adaptation of the infinitesimal method used to compute symmetry groups of differential equations [2, Chapter 7].

In some applications, finding equations or variational problems which do not have some desired classical Lie symmetries have remarkable importance. In this paper, we have focused on investigating a method to find such equations or problems. Our given method will be based on $\mu$-symmetries which was introduced by [3] to find Type I hidden symmetries. Hidden symmetries are defined as symmetries that are lost (Type I) or gained (Type II) as the order of an ODE is reduced or as the number of variable of a PDE is reduced [4]. Hidden symmetries are difficult to evaluate since there are no general direct methods for determining them. There are several approaches that we can use to investigate the hidden symmetries.

In 2001, Muriel and Romero introduced $\lambda$-symmetries to evaluate Type I hidden symmetries of ODEs [5]. Gaeta and Morando expanded this approach to scalar PDEs and PDEs systems as well as introducing a method for constructing equations without Lie point symmetries in $2004[3,6]$. These equations have no obvious order reduction (in ODE case) and variable reduction (in scalar PDEs case and PDEs systems) which can be reduced using $\mu$-symmetries. After a few months, in the subsequent paper [7], it was shown that $\mu$ symmetries are essentially equivalent (more precisely: locally, gauge equivalent) to standard symmetries (see especially [7], Theorem 1 and Example 1). The result has been also extended to the variational case in $[8,9]$.

As before, in this paper we will attempt to present a method for constructing variational problems without having a desired one-parameter transformation group as a variational symmetry. More details, this paper is organized as follows. In Section 2, briefly, the notation of $\mu$-symmetry is introduced in the line of $[3,6]$. Section 3 is devoted to applications of symmetry approach in variational problems and some basic concepts of variational calculus. Constructing equations without Lie symmetries and introducing their appropriate $\mu$-symmetries have been focused on in Section 4 . Section 5 can be considered as a main part of the paper which is devoted to construct variational problems without desired variational symmetries using results of Section 4 with Theorem 6 and assuming the obtained equations in the preceding section as the Euler-Lagrangian equations of some variational problems with necessary condition (Propositions 9 and $11)$. 


\section{2. $\mu$-Symmetry on Scalar PDEs and PDEs Systems}

As before, the starting point will be a discussion of some of the foundational results about $\mu$-symmetry. The reader can consult $[3,6]$ to gain complete information about these symmetries.

Let $\mu=\lambda_{i} d x_{i}$ be a horizontal one form on first order jet space $\left(J^{1} M, \pi, M\right)$ and compatible with contact structure $\varepsilon$ on $J^{k} M$ for $k \geq 2$. That is,

$$
d \mu \in J(\varepsilon)
$$

where $J(\varepsilon)$ is a Cartan ideal generated by contact structure.

Theorem 1 (see [3]). Condition (1) is equivalent to $D_{i} \lambda_{j}-$ $D_{j} \lambda_{i}=0$, where $D_{i}$ is total derivative with respect to $x_{i}$.

Let $X:=\sum_{i=1}^{p} \xi^{i}(x, u) \partial_{x_{i}}+\phi(x, u) \partial_{u}$ be an arbitrary vector field on total space $M . Y:=X+\Psi_{j} \partial u_{j}$ is defined as $\mu$-prolongation of $X$, on $k$ th order jet space $J^{k} M$, if its coefficient satisfies the following $\mu$-prolongation formula:

$$
\Psi_{J, i}=\left(D_{i}+\lambda_{i}\right) \Psi_{J}-u_{J, m}\left(D_{i}+\lambda_{i}\right) \xi^{m} \text {. }
$$

Remark 2. By setting $\mu=0$ in (2), we can gain ordinary prolongation of $X$. So, ordinary prolongation can be assumed as 0 -prolongation in $\mu$-prolongation framework.

In the following theorem, the connection between ordinary prolongation and $\mu$-prolongation will be considered.

Theorem 3 (see [3]). Let $X:=\sum_{i=1}^{p} \xi^{i} \partial_{x_{i}}+\Phi(x, u) \partial_{u}$ be a vector field on first order jet space $J^{1} M$; Let $Y=X+\Psi_{j} \partial_{u_{j}}$ and $X^{(k)}=X+\phi_{j} \partial_{u_{j}}$ be as $\mu$-prolongation and ordinary prolongation of $X$, respectively. Then, we have $\Psi_{J}=\Phi_{J}+F_{J}$, where $F_{J}$ satisfies the recursion relation (with $F_{0}=0$ ): $F_{J, i}=$ $\left(D_{i}+\lambda_{i}\right) F_{J}+\lambda_{i} D_{i} Q$; where, $Q$ is a Lie characteristic.

Note, this theorem provides an economic way of computing $\mu$-prolongation of $X$, if we already know its ordinary prolongation.

\section{Variational Problems and the Lie Standard Reduction Method}

In this section, we will point out some aspects of applications of symmetries method to variational problems. The reader can find more information in $[2,10,11]$. The variational problem is finding the extremals (maxima and/or minima) of a functional,

$$
\mathfrak{Q}_{\Omega}(u)=\int_{\Omega} L\left(x, u^{(n)}\right) d x, \quad \alpha=1,2, \ldots, q
$$

over some space of functions $u=f(x), x \in \Omega$. The integrand $L\left(x, u^{(n)}\right)$, which is a smooth differential function on the jet space $J^{(n)}$, is referred to as the Lagrangian of the variational problem (3). For such problems we can define the EulerLagrangian operators as

$$
E_{\alpha}=\sum_{J}(-D)_{J} \partial_{u_{J}^{\alpha}}, \quad \alpha=1,2, \ldots, q .
$$

Applications of the symmetry method on variational problems is motivated by the following theorem of the calculus of variational problems.

Theorem 4 (see [10]). The smooth extremals $u=f(x)$ of variational problem with the Lagrangian $L\left(x, u^{(n)}\right)$ must be satisfied in the systems of its related Euler-Lagrange equations:

$$
E_{\alpha}(L)=\Sigma_{J}(-D)_{J} \frac{\partial L}{\partial u_{J}^{\alpha}}
$$

The following theorem will be a cornerstone of our approach to the symmetry methods in variational problems.

Theorem 5 (see [2]). G is a variational symmetry of variational problem (3) if and only if it is a Lie symmetry of its EulerLagrangian equation (4).

\section{Euler-Lagrangian Equations without Desired Lie Symmetries}

Our given method mainly organized in two steps: first, we characterize equations (scalar PDEs) without desired Lie symmetries (this section); then, we find appropriate variational problems which have them as Euler-Lagrangian equations (next section).

To attain the pointed purpose of this section, we consider $X$ as a vector field on first order jet space $J^{1} M$ then determine general scalar PDEs which do not have $X$ as Lie symmetries, with admitting it as a $\mu$-symmetry (find more information in $[3,7])$.

Consider the scaling vector field $X=x \partial_{x}+t \partial_{t}+u \partial_{u}$. We have $Q=u-x u_{x}-t u_{t}$ for the Lie characteristic $[2,7]$. The corresponding invariant coordinates $(y, v)$ and the parametric coordinate $\sigma$ in $M=(x, t, u)$ can be chosen as $\sigma=x, y=t / x$ and $v=u / x$. The corresponding inverse change of variables is $x=\sigma, t=y \sigma, u=v \sigma$. Hence, the function $v=v(\sigma, y)$ is $X$-invariant if and only if $v_{\sigma}=0$. The partial derivations of $u$ express in the partial derivatives of $v=v(\sigma, y)$ as

$$
u_{x}=v+\sigma v_{\sigma}-y v_{y}, \quad u_{t}=v_{y} .
$$

The above can be inverted to give

$$
v_{y}=u_{t}, \quad v_{\sigma}=\frac{1}{x}\left[u_{x}+y u_{t}-\frac{u}{x}\right] .
$$

Similar to the above, we have the following expressions for second order derivatives:

$$
\begin{aligned}
& u_{x x}=2 v_{\sigma}+\sigma v_{\sigma \sigma}-y v_{\sigma y}+\frac{y^{2}}{\sigma} v_{y y}, \\
& u_{x t}=v_{\sigma y}-\frac{y}{\sigma} v_{y y}, \quad u_{t t}=\frac{1}{\sigma} v_{y y} .
\end{aligned}
$$


The central object in this computation is a horizontal oneform $\mu$ on one ordered jet space $J^{(1)} M$. Here, since the space of independent variables is two dimensional, we have $\mu=$ $\lambda d x+\tau d t$. Let us come to the second $\mu$-prolongation of $X$. For this computation, we can use (2) or the recursion relation in Theorem 3. Hence if we show $\mu$-prolongation of $X$ as

$$
Y=X+\Psi^{x} \partial_{u_{x}}+\Psi^{t} \partial_{u_{t}}+\Psi^{x x} \partial_{u_{x x}}+\Psi^{x t} \partial_{u_{x t}}+\Psi^{t t} \partial_{u_{t t}},
$$

then, we have

$$
\begin{gathered}
\Psi^{x}=\lambda Q, \quad \Psi^{t}=\tau Q \\
\Psi^{x x}=-u_{x x}+2 \lambda\left(D_{x} Q\right)+\left[\lambda^{2}+\left(D_{x} \lambda\right)\right] Q \\
\Psi^{x t}=-u_{x t}+\left[\lambda\left(D_{t} Q\right)+\tau\left(D_{x} Q\right)\right] \\
+\left(\frac{1}{2}\right)\left[2 \lambda \tau+\left(D_{t} \lambda\right)+\left(D_{x} \tau\right)\right] Q \\
\Psi^{t t}=-u_{t t}+2 \tau\left(D_{t} Q\right)+\left[\tau^{2}+\left(D_{t} \tau\right)\right] Q .
\end{gathered}
$$

We will now consider the simplest nontrivial choice for $\mu$; that is, $\mu=\lambda d x$, with $\lambda$ a real constant. By substituting this $\mu$ in (10), we find

$$
\begin{gathered}
\Psi^{x}=\lambda\left(u-x u_{x}-t u_{t}\right), \quad \Psi^{t}=0, \\
\Psi^{x x}=-u_{x x}-2 \lambda\left(x u_{x x}+t u_{x t}\right)+\lambda^{2}\left(u-x u_{x}-t u_{t}\right), \\
\Psi^{x t}=-u_{x t}-\lambda\left(x u_{x t}+t u_{t t}\right), \quad \Psi^{t t}=-u_{t t} .
\end{gathered}
$$

By taking $(y, v)$ as invariants of order zero, $\left(\xi_{1}, \xi_{2}\right)$ of order one, and $\left(\eta_{1}, \eta_{2}, \eta_{3}\right)$ of order two, we have

$$
\begin{gathered}
y=\frac{t}{x}, \quad v=\frac{u}{x}, \quad \xi_{1}=\left(\frac{u}{x}-\frac{t u_{t}}{x}-u_{x}\right) e^{\lambda x} \\
\xi_{2}=u_{t}, \quad \eta_{1}=x u_{t t}, \quad \eta_{2}=\left(x^{2} u_{x t}+x t u_{t t}\right) e^{\lambda x}, \\
\eta_{3}=\left(\frac{1}{x}\right)\left[(1-\lambda x)\left(u-x u_{x}\right)+\lambda x t u_{t}\right. \\
\left.+x^{2} u_{x x}+x t u_{x t}+t^{2} u_{t t}\right] e^{2 \lambda x}
\end{gathered}
$$

Theorem 6. Consider the equation $\Delta:=F\left(y, v, \xi_{1}, \xi_{2}, \eta_{1}\right.$, $\eta_{2}, \eta_{3}$ ) with $F$ as an arbitrary smooth function of its arguments. Let $\lambda$ be a real constant. Then, we have the following.

(i) Equation $\Delta$ admits the vector field $X$ as a $\mu$-symmetry with $\mu=\lambda d x$.

(ii) $\operatorname{For}\left(\partial F / \partial \xi_{1}\right)^{2}+\left(\partial F / \partial \eta_{2}\right)^{2}+\left(\partial F / \partial \eta_{3}\right)^{2} \neq 0, X$ is not an ordinary symmetry of $\Delta$.

(iii) The $\mu$-symmetry-reduced equation, providing $X$-invariant solutions to $\Delta$, is given by

$$
H\left[y, v, \xi_{2}, \eta_{1}\right]:=F\left[y, v, 0, \xi_{2}, \eta_{1}, 0,0\right]=0 .
$$

Proof. (i) Based on the Lie point symmetry method, a PDE admits $X$ as $\mu$-symmetry when we can rewrite it in terms of $X$-invariants [2]. Hence, equation $\Delta$ admits $X$ as $\mu$-symmetry with $\mu=\lambda d x$.

(ii) Using (12), we can conclude that, in solution space, the arguments $\xi_{1}, \eta_{2}$ and $\eta_{3}$ depend on $\mu$. So, if $F$ depends on this argument, then $X$ is not an ordinary symmetry of $\Delta$.

(iii) Finally, note that restriction to solution space does not change the form of $\xi_{2}$ and $\eta_{1}$. From the above explicit expression of the invariants, we have that $\xi_{1}=e^{\lambda x} Q / x, \eta_{2}=$ $-x e^{\lambda x}\left(D_{t} Q\right)$, and $\eta_{3}=\left(e^{2 \lambda x} / x\right)\left[(1-\lambda x) Q-2 t\left(D_{t} Q\right)-\right.$ $\left.x\left(D_{x} Q\right)\right]$. Therefore, on solution space we have $\xi_{1}=\eta_{2}=$ $\eta_{3}=0$, and (13) represents indeed the reduced equation for $\Delta$ in the space of $X$-invariant functions. This proves point (iii) and hence completes the proof.

We would like to note that, as claimed by (13), the reduced equation corresponds to the one which would be obtained by the standard symmetry reduction (under $X$ ) of the equation $F\left[y, v, \widehat{\xi}_{1}, \xi_{2}, \eta_{1}, \widehat{\eta}_{2}, \widehat{\eta}_{3}\right]=0$ to solution space, where $\widehat{\xi}_{1}$ is obtained from $\xi_{1}$ by setting $\lambda=0$, and the like for $\widehat{\eta}_{2}$ and $\widehat{\eta}_{3}$.

Finally, let us discuss a completely concrete example; albeit, the procedure here is completely analogous to the one to be followed for standard symmetries. Consider equation $\Delta:=\eta_{1}-\eta_{2}=0$. This is written as

$$
x u_{t t}-\left(x^{2} u_{x t}+x t u_{t t}\right) e^{\lambda x}=0,
$$

in the original coordinates; in the adapted ones it reads

$$
v_{y y}-\sigma^{2} v_{\sigma y} e^{\sigma \lambda}=0
$$

The corresponding reduced equation is

$$
v_{y y}=0 \text {, }
$$

and the general solution to this is $v(y)=c_{1}+c_{2} y$, where $c_{i}$ are real constants. Going back to the original coordinates, the corresponding solutions are $u(x, t)=c_{1} x+c_{2} t$.

Remark 7. Note, unlike the Lie method, we focus on solutions which have not admitted the desired Lie symmetries. For instance, here, beside finding the solution $u(x, t)=c_{1} x+c_{2} t$ for (14), we found that for some $s$, this does not remain again a solution under scaling transformation $(x, t, u) \mapsto$ $\left(e^{s} x, e^{s} t, e^{s} u\right)$.

\section{5. $\mu$-Symmetry on Variational Problems}

In the calculus of variations, the problem of characterizing systems of differential equations which are the EulerLagrange equations for some variational problems is known as the inverse problem $[7,8]$. There are different approaches to perform the inverse problem $[8,9]$. Here, in order to keep the scope manageable, we use the direct method.

In this section, applying the preceding results, we construct an example of a variational problem which its EulerLagrange equation has no desired Lie symmetries. For this 
purpose, first, we assume that $\Delta$ is an equation without desired Lie symmetry (here, scaling symmetry); then, we find appropriate variational problems which have equation $\Delta$ as a Euler-Lagrangian equation. Also, in Proposition 11, we will introduce the class of variational problems without having any nontrivial Lie variational symmetries.

Example 8. Consider this equation

$$
x u_{t t}-e^{x}\left(x^{2} u_{x t}+x t u_{t t}\right)=0,
$$

it can be written as: $\eta_{1}-\eta_{2}=0$, using given expressions in (12) with $\lambda=1$. Based on Theorem 6 this equation has scaling symmetry: $(x, t, u) \mapsto\left(e^{s} x, e^{s} t, e^{s} u\right)$ as $\mu$-symmetry with $\mu=d x$, and this transformation is not its Lie symmetry. Let it be an Euler-Lagrange equation for some second-order variational problem; so, from (4) we have

$$
\begin{aligned}
\frac{\partial L}{\partial u}- & D_{x} \frac{\partial L}{\partial u_{x}}-D_{t} \frac{\partial L}{\partial u_{t}}+D_{x}^{2} \frac{\partial L}{\partial u_{x x}}+D_{x} D_{t} \frac{\partial L}{\partial u_{x t}}+D_{t}^{2} \frac{\partial L}{\partial u_{t t}} \\
& =x t u_{t t}-e^{x}\left(x^{2} u_{x t}+x t u_{t t}\right) .
\end{aligned}
$$

By solving this equation, we have

$$
\begin{aligned}
& L_{1}\left(x, t, u, u_{x}, u_{t}, u_{x x}, u_{x t}, u_{t t}\right) \\
& \quad=\frac{1}{2} u\left(x t u_{t t}-e^{x}\left(x^{2} u_{x t}+x t u_{t t}\right)\right) .
\end{aligned}
$$

As a result of the above and using Theorem 5 and Corollary 7.4 in [2], we can find the following.

Proposition 9. Any variational problem with the Lagrangian $\widehat{L}=L_{1}+$ Div $\xi$ for arbitrary smooth function $\xi$ does not admit scaling transformation $\left(e^{s} x, e^{s} t, e^{s} u\right)$ as a variational symmetry. Moreover, this transformation is a $\mu$-symmetry of its Euler-Lagrangian equation with $\mu=d x$.

Example 10. Consider the equation

$$
u_{x x}+\frac{x^{2}}{4 u^{3}}+u+\frac{1}{2 u}=0 \text {. }
$$

This equation appears in Theorem 4.3 of [5] as an equation which can be integrated using the $\lambda$-symmetry method with $\lambda=x / u^{2}$ and $X=u \partial_{u}$ but lacks nontrivial symmetries. Let it be an Euler-Lagrange equation for some second-order variational problem; so, from (4) we have

$$
\frac{\partial L}{\partial u}-D_{x} \frac{\partial L}{\partial u_{x}}=u_{x x}+\frac{x^{2}}{4 u^{3}}+u+\frac{1}{2 u},
$$

by solving (21), we find the following Lagrangian:

$$
L_{2}\left(x, u, u_{x}\right)=\frac{1}{2}\left(u^{2} u_{x}+u^{2}+\ln u-u_{x}^{2}-\frac{x^{2}}{4 u^{2}}\right) .
$$

Using (Theorem 5) and Corollary 7.4 of [2], the following will hold.
Proposition 11. Any variational problem with the Lagrangian $\widehat{L}=L_{2}+\operatorname{Div} \xi$ for arbitrary smooth function $\xi$ has no nontrivial variational Lie symmetries, where its Euler-Lagrangian equation has $X=u \partial_{u}$ as a $\lambda$-symmetry with $\lambda=x / u^{2}$.

\section{Conclusions}

In this paper, first, we construct some Euler-Lagrange equations with no desired symmetries as well as introducing appropriate $\mu$-symmetries to reducing them; next, we find their related variational problems without having desired variational symmetries. Indeed, in Propositions 9 and 11, we introduced two class of variational problems which have no scaling variational symmetry and nontrivial Lie symmetries, respectively.

\section{Acknowledgment}

The authors would like to thank the referees for constructive suggestions and helpful discussions which have materially improved the presentation of the paper.

\section{References}

[1] S. Lie, "Uber integralinvarianten und ihre Verwertung fur die Theorie der Differentialgleichungen," in Gesammelte Abhandlungen, vol. 6, pp. 664-701, B.G. Teubner, Leipzig, Germany, 1927.

[2] P. J. Olver, Equivalence, Invariants, and Symmetry, Cambridge University Press, Cambridge, UK, 1995.

[3] G. Gaeta and P. Morando, "On the geometry of lambdasymmetries and PDE reduction," Journal of Physics A, vol. 37, no. 27, pp. 6955-6975, 2004.

[4] B. Abraham-Shrauner, "Hidden symmetries and nonlocal group generators for ordinary differential equations," IMA Journal of Applied Mathematics, vol. 56, no. 3, pp. 235-252, 1996.

[5] C. Muriel and J. L. Romero, "New methods of reduction for ordinary differential equations," IMA Journal of Applied Mathematics, vol. 66, no. 2, pp. 111-125, 2001.

[6] G. Gaeta and P. Morando, "PDEs reduction and $\lambda$-symmetries," Note di Matematica, vol. 23, no. 2, pp. 33-73, 2004/05.

[7] G. Cicogna, G. Gaeta, and P. Morando, "On the relation between standard and $\mu$-symmetries for PDEs," Journal of Physics $A$, vol. 37, no. 40, pp. 9467-9486, 2004.

[8] C. Muriel, J. L. Romero, and P. J. Olver, "Variational $C^{\infty}$ symmetries and Euler-Lagrange equations," Journal of Differential Equations, vol. 222, no. 1, pp. 164-184, 2006.

[9] G. Cicogna and G. Gaeta, "Noether theorem for $\mu$-symmetries," Journal of Physics A, vol. 40, no. 39, pp. 11899-11921, 2007.

[10] B. van Brunt, The Calculus of Variations, Springer, 2003.

[11] P. J. Olver, Applications of Lie Groups to Differential Equations, vol. 107, Springer, New York, NY, USA, 1986. 


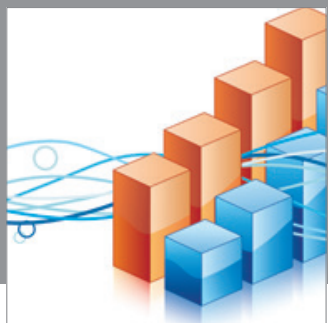

Advances in

Operations Research

mansans

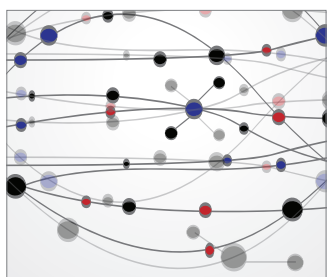

The Scientific World Journal
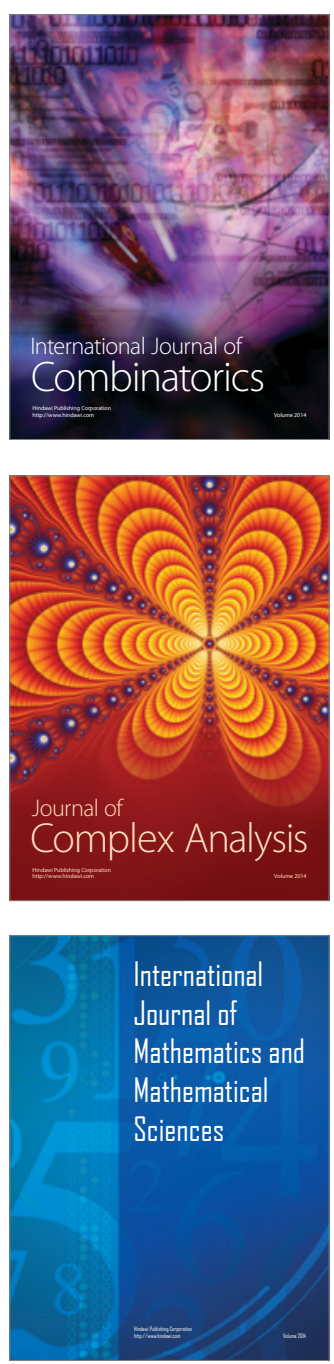
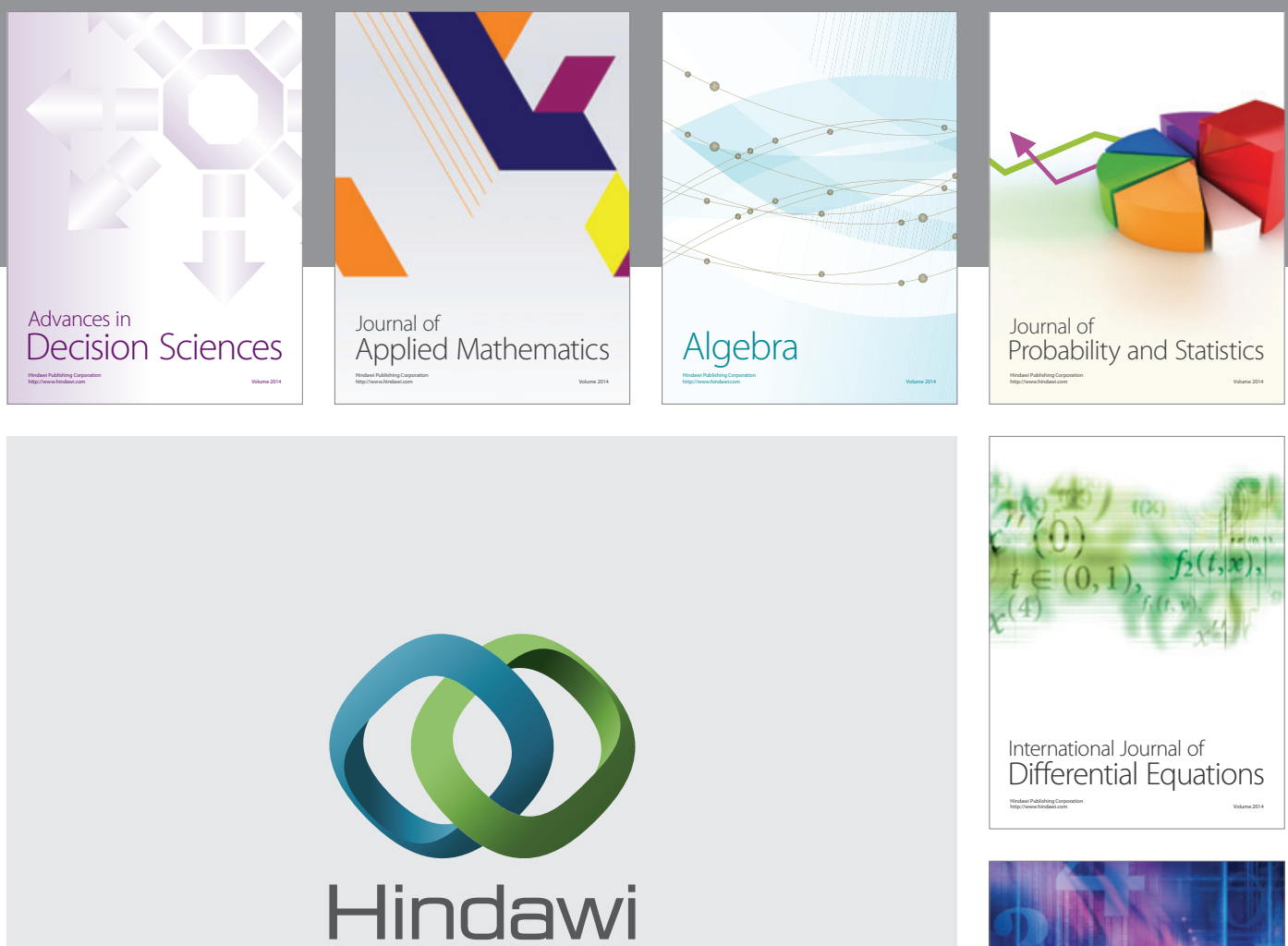

Submit your manuscripts at http://www.hindawi.com
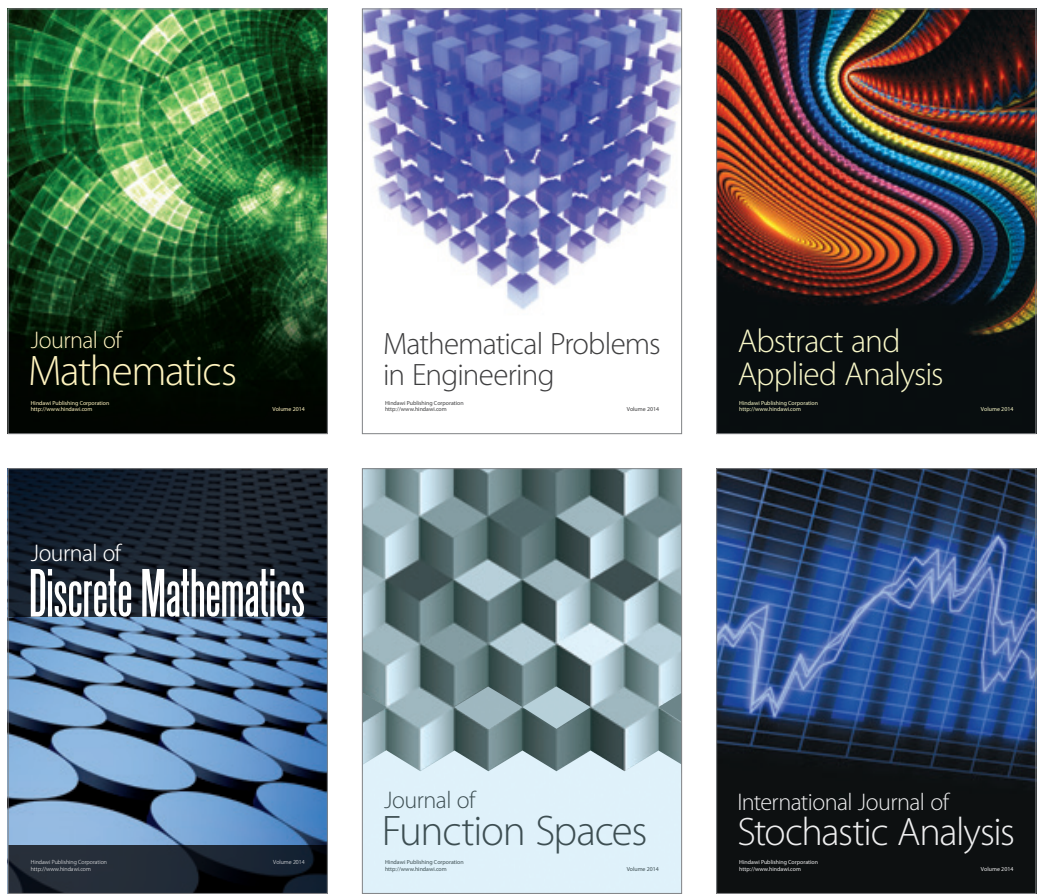

Journal of

Function Spaces

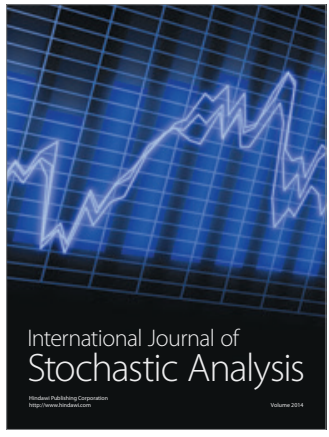

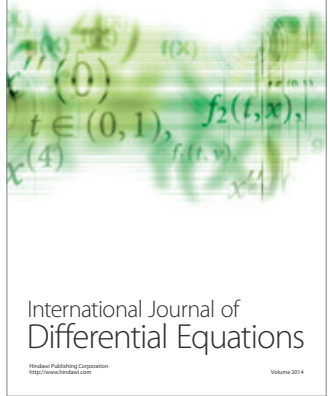
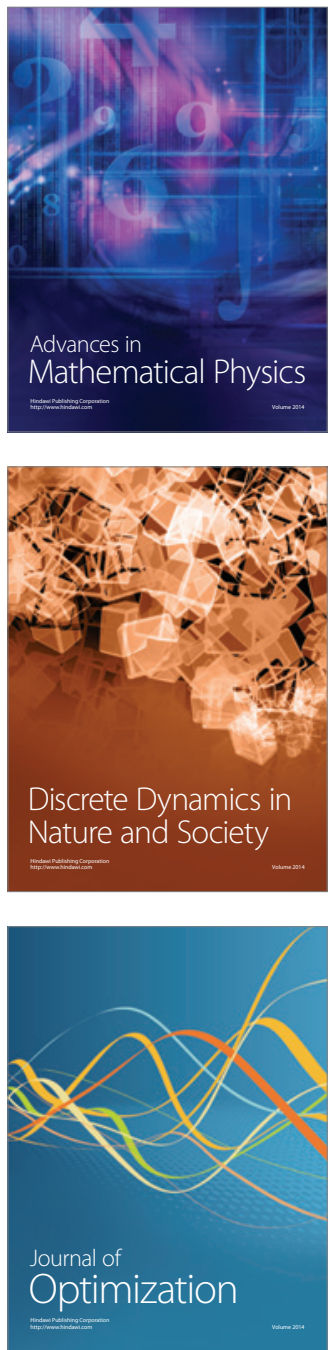